\title{
Assessment of a screening test to identify Lyme disease risk
}

\author{
Ogden $\mathrm{NH}^{1^{*}}$, Koffi JK${ }^{1}$, Lindsay $\mathrm{LR}^{2}$ \\ ${ }^{1}$ Centre for Food-borne, Environmental and Zoonotic Infectious Diseases, Public Health Agency of Canada, \\ Saint-Hyacinthe, Quebec \\ ${ }^{2}$ National Microbiology Laboratory, Public Health Agency of Canada, Winnipeg, Manitoba \\ * Corresponding author: nicholas.ogden@phac-aspc.gc.ca
}

\section{Abstract}

Background: Lyme disease is emerging in eastern and central Canada due to the spread of the tick vector Ixodes scapularis. Currently, the test to establish Lyme disease-endemic areas requires multiple site visits and multiple sampling methods, and is consequently complex, time-consuming, and resource-expensive.

Objective: To assess the capacity of drag sampling alone as a screening technique to identify areas of Lyme disease risk.

Method: We conducted a retrospective analysis of field surveillance data obtained at 100 site visits in 2007 and 2008 in southern Quebec.

Outcome: Drag sampling used alone had $50 \%$ sensitivity but $86 \%$ specificity to identify early-established $I$. scapularis populations. Ticks were found throughout the period May to October.

Conclusion: One site visit of drag sampling of three person-hours between May and October may be sufficient to identify a Lyme disease risk location. This information can be used by public health professionals to develop public health responses and by medical practitioners to assist in the clinical diagnosis of Lyme disease.

\section{Introduction}

Lyme disease in North America is caused by the spirochete Borrelia burgdorferi sensu stricto (henceforth referred to as Borrelia burgdorferi), which is transmitted by the tick vector Ixodes scapularis in eastern and central North America (1). The geographic range of $I$. scapularis is spreading from the United States into eastern and central Canadian provinces (2), due to dispersal of ticks by songbirds during spring migration, and enhanced environmental suitability for the tick with a changing climate (3). Generally, where the ticks have become established, Lyme disease risk follows (4).

Critical to the management of the expanding risk of Lyme disease in Canada is identifying where the ticks have become established and are transmitting $B$. burgdorferi among their wildlife hosts (5). This is important both to inform public health activities by identifying the population at risk, and to aid in the clinical diagnosis of Lyme disease and the interpretation of laboratory tests (5-6).

The occurrence of possible new locations of Lyme disease risk can be signaled by the occurrence of Lyme disease cases in these locations, or by information from passive tick surveillance $(7,3)$. The current gold standard to confirm the presence of Lyme disease risk (i.e. to identify a Lyme disease-endemic area) in these areas, or in prospective studies, requires considerable human and financial resources over a two-year period. Endemic areas for the tick vectors are defined as a contiguous sampling area where "all three stages larva, nymph and adult are present .... on resident animals or in the environment for at least 2 consecutive years" (7), Lyme disease-endemic areas are those where the tick vectors are endemic and where there is evidence the ticks are transmitting $B$. burgdorferi among reservoir hosts (7). Identification of these areas requires drag sampling and the capture and testing of a minimum of 30 wild rodents. 
Drag sampling involves trailing a $1-\mathrm{m}^{2}$ flannel through the habitat to collect unfed ticks that are host-seeking on the woodland floor. Rodents are captured in live traps and examined for ticks. Wild rodents are natural hosts of larval and nymphal ticks as well as being reservoir hosts for $B$. burgdorferi. Ticks and blood and tissue samples from captured rodents are tested for evidence of $B$. burgdorferi infection by polymerase chain reaction (PCR), serology, and/or culture. Although this method has a high specificity and sensitivity to detect infected ticks and $B$. burgdorferi, it is time consuming. It requires multiple site visits to place traps and then open them to sample rodents the following day. This process is usually repeated three times a year for two years. This is challenging both logistically and in terms of human and financial resource needs, is limited by the availability of trained field operatives and may limit the amount of field surveillance that can be conducted by provincial and municipal public health organizations.

In the United States, the mainstay of field surveillance is drag sampling alone, based on the observation that the numbers of infected ticks collected by drag sampling correlates with risk to humans (8). It is also known that different tick stages are active at different times of the year, and tick abundance overall can vary from one year to the next, particularly in emerging risk areas (10).

The objective of this study was to assess whether drag sampling alone may be sufficient as a screening technique to identify $I$. scapularis populations and Lyme disease risk in Canada and whether this may be affected by the time of year sampling occurs.

\section{Methods}

To assess drag sampling alone as a screening technique to identify Lyme disease risk, we retrospectively analyzed data from previous field studies carried out at 71 individual sites in southern Quebec in 2007 and 2008. $(3,9)$. Sites were selected to ensure the habitat was suitable for I. scapularis and to minimize intersite variations in habitat. Variations in presence and numbers of $I$. scapularis ticks was mostly determined by temperature conditions at the sites (3). Some of the 71 sites were visited up to four times over the period from 2007 to 2008, so there were data from 100 site visits. At each site visit, the presence of $I$. scapularis was determined by drag sampling the environment (with an effort of three person-hours per site visit), and by examination of captured rodents for feeding ticks. At each site visit, 15 mice were captured, giving 95\% probability of detecting a tickinfested rodent if the true prevalence is $20 \%$. This was lower than the 30 mice recommended by guidelines developed by Health Canada in 1991 (to give 95\% probability of detecting an infested mouse if the true prevalence was 10\%), as the objective in the 1991 guidelines was to confirm the absence of ticks (7). The species of all collected ticks was determined using standard tick identification keys (3). All identification of ticks and laboratory analyses for $B$. burgdorferi infection in samples collected from these sites were conducted at the Public Health Agency of Canada's National Microbiology Laboratory.

For each site visit, the identification of $I$. scapularis on rodents and by drag sampling were considered as binary data (i.e. either present or absent). To assess the sensitivity and specificity of drag sampling, the presence or absence of ticks by drag sampling was compared with the presence or absence of ticks in the captured rodents. Sensitivity of drag sampling was calculated as the proportion of site visits where ticks were found by drag sampling and there were also I. scapularis-infested rodents. Specificity was the proportion of the site visits where ticks were absent by drag sampling and there were no $I$. scapularis-infested rodents. Ticks collected by drag sampling during site visits where there were no I. scapularis-infested rodents could be considered to be adventitious ticks, i.e. ticks carried into the site by animal or bird hosts that acquired the ticks in other locations.

To explore whether the season or year of site visits had an impact on the ability of drag sampling to detect ticks, two logistic regression models were created in which site identification number was a random effect (as some sites were visited multiple times). In the first model, we explored whether there were seasonal variations in the capacity of examination of captured rodents to detect $I$. scapularis on a site. In this model, the outcome variable was presence of $I$. scapularis as determined by examination of captured rodents, and the explanatory variables were year of sampling and season of sampling (season 1 being May to June, season 2 being July to August, and season 3 being September to October). In the second model, we explored whether the season and year of sampling were associated with variations in the capacity of drag sampling to predict the presence of $I$. scapularis 
on rodents. Interactions amongst drag-sampling results and season were also explored. All analyses were conducted in Stata SE 11.0 (StataCorp, College Station, Tx) and the threshold for significance was $\mathrm{P}<0.05$.

\section{Results}

A total of one hundred site visits were conducted at 71 sites over a two-year period. At all the sites visited, the density of ticks and $B$. burgdorferi infection prevalence were low (3), which was consistent with early stage of establishment of the tick and $B$. burgdorferi transmission cycles $(10,11)$. Eleven of the sites at which $I$. scapularis were identified at visits in 2007 were revisited in 2008 at least once. At the 2008 site visits, two or more stages of I. scapularis were detected at all of the sites, supporting the idea that these sites did not just have adventitious ticks as they contained reproducing populations of I. scapularis (3).

Of the 58 site visits at which I. scapularis were collected from captured rodents, 29 also yielded $I$. scapularis by drag sampling, so the sensitivity of drag sampling was $50 \%$ (Table 1). Of the 42 site visits at which no $I$. scapularis were found on rodents, 36 yielded no I. scapularis by drag sampling, so the specificity of drag sampling was $86 \%$ (36/42). However, on two of the sites on which drags were positive, rodent testing was negative, but ticks were found on rodents at previous or subsequent visits, suggesting that in these two instances, ticks collected by drag sampling were not adventitious ticks.

Table 1: Sensitivity and specificity of drag sampling alone to detect I. scapularis feeding on captured rodents ( $\mathrm{n}=100$ site visits)

\begin{tabular}{|l|l|l|}
\hline & $\begin{array}{l}\text { I. scapularis ticks detected on rodents } \\
\mathbf{n}=58\end{array}$ & $\begin{array}{l}\boldsymbol{I} \text {. scapularis ticks not detected on } \\
\text { rodents } \mathbf{n}=42\end{array}$ \\
\hline $\begin{array}{l}\text { Drag sampling was } \\
\text { POSITIVE }\end{array}$ & $29 / 58=50 \%$ sensitivity & $6 / 42$ \\
\hline $\begin{array}{l}\text { Drag sampling was } \\
\text { NEGATIVE }\end{array}$ & $29 / 58$ & $36 / 42=86 \%$ specificity \\
\hline
\end{tabular}

The proportion of site visits at which I. scapularis were collected from captured rodents was significantly greater in 2008 than 2007 (odds ratio $[\mathrm{OR}]=10.7,95 \% \mathrm{Cl} 1.01-105.6, \mathrm{P}<0.05$ ), but there was no significant variation amongst seasons $\left(x^{2}=2.4, \mathrm{df}=2, \mathrm{P}>0.1\right)$. Similarly, the proportion of site visits at which $I$. scapularis were collected by drag sampling was significantly greater in 2008 than 2007 (OR $=6.4,95 \% \mathrm{Cl} 2.23-18.36, \mathrm{P}<0.05)$, but there was no significant variation among seasons $\left(x^{2}=2.6\right.$, df $\left.=2, P>0.1\right)$. The seasonal activity of the different stages of $I$. scapularis on rodents and drags was also consistent with that expected of northeastern North America (i.e. adults active in spring and autumn, nymphs active in spring and early summer, and larvae active in late summer and early autumn) (3). When the detection of $I$. scapularis on drag sampling was included as an explanatory variable, it was significantly associated with the detection of $l$. scapularis on captured rodents $(O R=$ $9.48,95 \% \mathrm{Cl}=1.46-60.94$ ) but there were no significant interactions with the season or year.

\section{Discussion}

This analysis suggests that drag sampling has good specificity, but less sensitivity than examination of captured rodents to identify where Lyme disease may be acquired now or in the near future. A specificity of almost $90 \%$ suggests that if $I$. scapularis ticks are found by drag sampling, it is likely a risk area for Lyme disease. A sensitivity of $50 \%$ suggests that some sites with low densities of ticks will be missed by drag sampling alone. However, sites where ticks are present in rodents but undetected by drag sampling are most likely sites where tick populations and $B$. burgdorferi transmission cycles are at a very early stage of establishment, and pose a low level of Lyme disease risk to the public (10). When ticks were found by drag sampling and on rodents, ticks were also found at that site in the following year. Together, this means there is relatively high confidence that if an I. scapularis tick is found by drag sampling, then an emerging, self-sustaining, reproducing population of $I$. scapularis ticks likely is present at that location. 
The strength of this study is that our preliminary evidence suggests this simple field technique can offer a good indication of Lyme disease risk. This is consistent with American studies linking drag sampling results to human disease incidence (8). This is also consistent with what has occurred in Ontario and Quebec: as tick populations become more firmly established, tick density and tick infection prevalence will rise (4). Screening for Lyme disease risk by drag sampling alone is also relatively easy and cheap to operationalize and did not seem to be affected by seasonal variation.

A limitation of this study was using the positive rodent test as the comparator, versus the more comprehensive 1991 guidelines test for establishing a Lyme disease-endemic area. The rodent test was a good proxy but was not ideal, as evidenced by the two instances where drag sampling was positive but rodent test was negative. The assumption that these were adventitious ticks was not substantiated as ticks were found in rodents at those sites in subsequent years. Drag sampling also detects adult ticks active in spring and autumn, and these do not feed on rodents so would not be detected by rodent capture alone (10).

Another potential limitation of the study is that it did not assess the effectiveness of field training. Not all ticks, for example, are easily identifiable. Larvae active in late summer are smaller than spring-active nymphs and individually less likely to be found (10). Training of field staff to identify the very small immature ticks would be needed, but this training would be much easier to implement than training for capture, handling and/or anesthesia and examination of rodents.

Further study is warranted to explore the relationships between tick abundance and infection prevalence and incidence of human cases to more clearly be able to quantify the relationship between environmental measures of risk and disease risk. Also, replication of the study in different habitats and geographic locations (e.g. woodlands of British Columbia, Manitoba, and the Maritimes) would be prudent to explore and ensure the generalizability of the findings.

Drag sampling may be most useful in identifying the progression of areas at risk in provinces where Lyme disease risk has already been established. Once a Lyme disease-endemic area is confirmed, then the one-visit dragsampling method could be employed to identify areas of risk over a wider geographic area. However, it may be prudent for public health practitioners in provinces (such as Alberta, Saskatchewan, and Prince Edward Island) and the territories where I. scapularis or I. pacificus do not presently occur, to use the 1991 guidelines methodology recommended to detect Lyme disease-endemic areas (7) before using this screening test to assess emerging tick populations.

Our study suggests that a single drag-sampling visit may be a good screening test to detect Lyme disease risk locations to complement the 1991 guidelines approach to confirming a Lyme disease-endemic area. Studies assessing the effectiveness of teaching drag-sampling are indicated, as this would be a simple cost-effective way for local or regional public health to determine Lyme disease risk in their jurisdiction. This could then be used to alert public health and medical practitioners, and the public of a Lyme disease risk and enable a timelier implementation of public health interventions.

\section{References}

(1) Thompson C, Spielman A, Krause PJ. Coinfecting deer-associated zoonoses: Lyme disease, babesiosis, and ehrlichiosis. Clin Infect Dis. 2001;33:676-85.

(2) Ogden NH, Lindsay LR, Morshed M, Sockett PN, Artsob H. The emergence of Lyme disease in Canada. CMAJ. 2009;180:1221-4.

(3) Ogden NH, Bouchard C, Kurtenbach K, Margos G, Lindsay LR, Trudel L, et al. Active and passive surveillance and phylogenetic analysis of Borrelia burgdorferi elucidate the process of Lyme disease risk emergence in Canada. Environ Health Perspect. 2010;118:909-14.

(4) Ogden NH, Lindsay LR, Leighton P. Predicting the rate of invasion of the agent of Lyme disease, Borrelia burgdorferi in North America. J Appl Ecol. 2013;50:510-8. 
(5) Barker IK, Lindsay LR. Lyme borreliosis in Ontario: determining the risks. CMAJ. 2000;162:1573-4.

(6) Wormser GP, Dattwyler RJ, Shapiro ED, Halperin JJ, Steere AC, Klempner MS, et al. The clinical assessment, treatment, and prevention of Lyme disease, human granulocytic anaplasmosis, and babesiosis: Clinical practice guidelines by the Infectious Diseases Society of America. Clin Infect Dis 2006;43:1089-134.

(7) Health Canada. Consensus conference on Lyme disease. CMAJ. 1991;144:1627-32.

(8) Diuk-Wasser MA, Hoen AG, Cislo P, Brinkerhoff R, Hamer SA, Rowland M, et al. Human risk of infection with Borrelia burgdorferi, the Lyme disease agent, in eastern United States. Am J Trop Med Hyg. 2012;86:320-7.

(9) Ogden NH, St-Onge L, Barker IK, Brazeau S, Bigras-Poulin M, Charron DF, et al. Risk maps for range expansion of the Lyme disease vector, Ixodes scapularis, in Canada now and with climate change. Int J Health Geogr. 2008;7:24.

(10) Bouchard C, Beauchamp G, Nguon S, Trudel L, Milord F, Lindsay LR, et al. Associations between Ixodes scapularis ticks and small mammal hosts in a newly endemic zone in southeastern Canada: Implications for Borrelia burgdorferi transmission. Ticks Tick Borne Dis. 2011;2:183-90.

(11) Ogden NH, Mechai S, Margos G. Changing geographic ranges of ticks and tick-borne pathogens: drivers, mechanisms and consequences for pathogen diversity. Front Cell Infect Microbiol. 2013;3:46.

\section{Acknowledgements}

We acknowledge all our co-authors on the previous studies $(3,9)$ that provided the data analyzed here.

\section{Conflict of interest}

There are no conflicts of interest to declare.

\section{Funding}

The field work of this study was funded by the Public Health Agency of Canada and the Institut de santé publique du Québec. 